\title{
Towards single cell spectroscopy and refractometry in microfluidic chip platforms
}

\author{
J. B. Tillak ${ }^{\mathrm{a}}$, I. Bernacka-Wojcik ${ }^{\mathrm{b}}$, D. Barata, ${ }^{\mathrm{b}, \mathrm{c}}$, P. A. S. Jorge ${ }^{\mathrm{a}^{*}}$, H. Águas ${ }^{\mathrm{b}}$, A. G. Oliva ${ }^{\mathrm{c}}$ \\ ${ }^{a}$ INESC Porto, Rua do Campo Alegre, 687, 4169-007 Porto, Portugal \\ ${ }^{\mathrm{b}}$ Departamento de Ciência dos Materiais, Campus de Caparica, 2829-516 Caparica, Portugal \\ ${ }^{c}$ Biomolecular Diagnostic Laboratory, ITQB-UNL/IBET, Av. da República - Qta do Marquês, Apartado 12, 2781-901 \\ Oeiras, Portugal
}

\begin{abstract}
This paper evaluates various strategies proposed for single cell refractometry and spectroscopy using fiber optic sensors and microfluidic chips. Details concerning design, fabrication and characterization of the chips will be addressed. Preliminary results obtained with alternative on-chip configurations using combination of fiber Bragg gratings with mirrored single mode and multimode fibers will be presented indicating the possibility of performing simultaneous assessment of cellular refractive index and absorption properties.
\end{abstract}

Keywords: single cell analysis, microfluidics, spectroscopy, Fabry-Perot cavity, optical fiber sensors

\section{INTRODUCTION}

Spectroscopy and refractometry are commonly used in chemical and biochemical systems due to its numerous advantages like real-time and non-intrusive measurements, good reliability and sensitivity. Detection through optical absorption and fluorescence spectroscopy are some of the most widely used methods in common biosensing systems [1]. Near infra red (NIR) interferometry, on the other hand, can give complementary information allowing on-line monitoring of reactions thought detection of refractive index changes induced by the analyte [2]. Combination of those methods may extend the spectrum of information about investigated samples and allow better accuracy of results. For instance, detection of significant variations of refractive index of living cells can be useful in sensing of e.g. intraerythrocyte parasites or cancer forms $[3,4]$, while monitoring its color changes can expand the spectrum of applications allowing better determination of the stage of the disease, especially in the case of advanced cancerous forms or in a parasitic invasion, where the classical single-wave spectroscopy reveals reduced resolution.

Since the concept of microfluidic chip was proposed [5], the development of miniaturized integrated systems on the chip platform has gained huge popularity in analytical chemistry. In comparison with standard analytical methods, microfluidic devices offer the advantages of faster sample analysis, higher throughput, reduced reagent use, automation of complex bioanalytical processes and ease of use, all of which are associated with decreased cost $[6,7]$.

For the fabrication of the microfluidic platform various approaches are possible and many alternatives are described in literature [8]. The use of a microstructured layout onto glass support, closed by a polymeric block (e.g. PDMS), allows practical and easy mounting and cleaning of the chip [9]. SU-8, an epoxy-based negative photoresist, is one of the most used materials for the fabrication of high aspect ratio (the ratio of height to the width) microfluidics due to its good chemical, mechanical and thermal properties [10].

Recently many microfluidic systems have been explored for cell diagnosis and characterization. Cell refractive index was measured using various approaches, mostly based in interferometric analysis. Setups varied from optofluidic cavity between mirrored glass plates, to more advanced microstructures using cell holders or optical trapping. In this context, fiber Bragg grating (FBG) based cavities are interesting alternatives for interferometric on-chip analysis [11-13].

This paper describes a microscale setup for simultaneous NIR interferometry and absorption or fluorescence spectroscopy based on an SU-8 microfluidic platform with embedded fiber optic sensors. Several strategies were evaluated in order to simultaneously detect refractive index and absorption properties of cells. Preliminary results of measurements performed with sample solutions and the proposed microchip configurations are presented.

* pedro.jorge@inescporto.pt; phone +351 22040 2301; www.inescporto.pt

International Conference on Applications of Optics and Photonics, edited by Manuel F. M. Costa, Proc. of SPIE Vol. 8001, 800112 · @ 2011 SPIE · CCC code: 0277-786X/11/\$18 · doi: 10.1117/12.892180 


\section{CHIP DESIGN AND WORKING PRINCIPLES}

\subsection{Chip design}

The microfluidic chip was fabricated by photolithographic process of SU-8 layers over glass substrates. The microchip structure is presented in figure 1a. Each chip consists of a $20 \mu \mathrm{m}$ wide central micro-channel connected with a network of 3 inlets and 3 outlets for sample delivery and processing. Depending on the final configuration, one, two or more grooves can be placed perpendicularly to the channel for insertion of optical fibers. The width and depth of the fiber insertion grooves was around $127 \mu \mathrm{m}$, allowing optical fibers to be accurately fixed and aligned enabling light to be transmitted through the fluid. Each of the fiber, depending on its characteristics (multimode, single mode) can be used for delivery or collection of light in the visible or NIR range, enabling spectroscopy or interferometric measurements to be performed.

Distinct interfaces between the optical fiber and the micro channel are being tested. Figure 2 shows two of the possibilities implemented: (b) channel and fiber with SU-8 separation walls (c) or with SU-8 separation clips. Also a chip configuration with direct contact of the fiber with the channel has been fabricated.

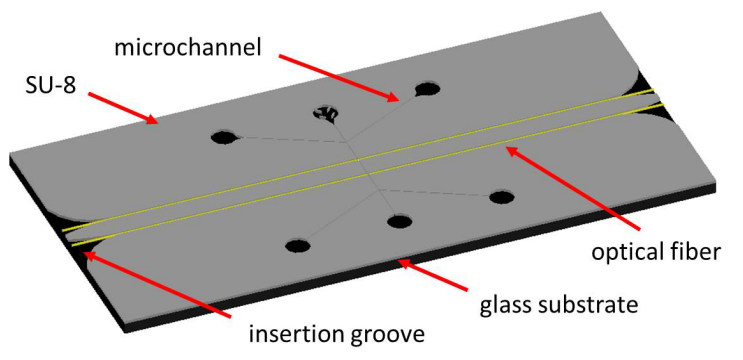

(a)

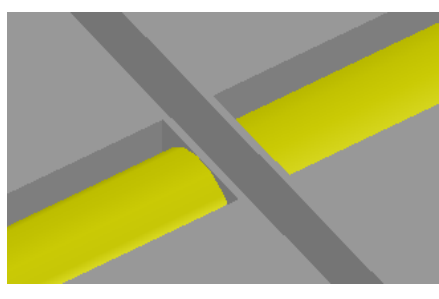

(b)

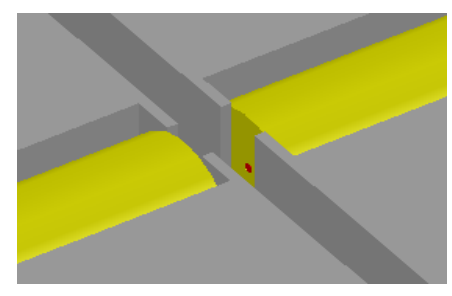

(c)

Figure. 1. (a) Schematic representation of the SU-8 micro fluidic chip with inserted optical fibers. Detail of the tested interfaces between micro channel and optical fibers: (b) with a SU-8 separation wall; (c) with SU-8 clips.

\subsection{Optical sensors}

With such platform as a starting point, distinct sensing configurations can then be explored using different combinations of single mode (SM) or multimode (MM) fibers, fiber Bragg gratings and mirrors inserted into the microfluidic chip. In figure 2, a generic combination of such on-chip fiber optic devices is presented.

For instance, if two identical FBG inscribed in SM fibers are placed a distance $L f$ apart as depicted in figure 2, a Fabry Perot (FP) cavity can be obtained as described by Chin et al. [13]. If, on the other hand, one of the fiber tips is mirrored, only one FBG becomes necessary to obtain the same FP cavity. In such case, the mirrored fiber can be a MM fiber and, if the mirror is just partially reflecting, light can be collected by a spectrometer, enabling the measurement of the absorption/reflectance spectra of the samples flowing in the intercalated microchannel, using standard microscope illumination.

Such configuration will enable the combination of simultaneous detection of refractive index and absorption and scattering properties of the flowing samples. The cell refractive index can be measured in the NIR range $(\lambda=1550 \mathrm{~nm})$ using SM fiber, an FBG and a mirrored fiber across the flow channel. Shifts induced in the measured interferometric spectra have been shown to be correlated with the cell optical path $[12,13]$.

Reflectance spectra of the cells, on the other hand, can be acquired using the partially mirrored MM fiber that collects the visible $(450 \mathrm{~nm}-800 \mathrm{~nm})$ range radiation emitted by the microscope illumination and is scattered /absorbed by the passing cells. Standard CCD spectrometer can be used to acquire the signal with the necessary spectral discrimination.

Experiments on Plasmodium falciparum parasited erythrocyte showed significant changes in refractive index when comparing healthy and infected cells, that could be as high 0,03 RIU [4]. On the other hand, a detailed analysis of the 
reflectance spectra of healthy and Escherichia. coli. infected blood samples, framed with an adequate theoretical model, enabled the quantitative interpretation of the acquired visible-NIR spectra and its correlation with the infectious state of the cells [14].

In this context, the proposed configuration is an excellent starting point for a more complete and accurate characterization and diagnosis of living cells as it will enable the simultaneous application of two distinct optical sensing mechanisms.

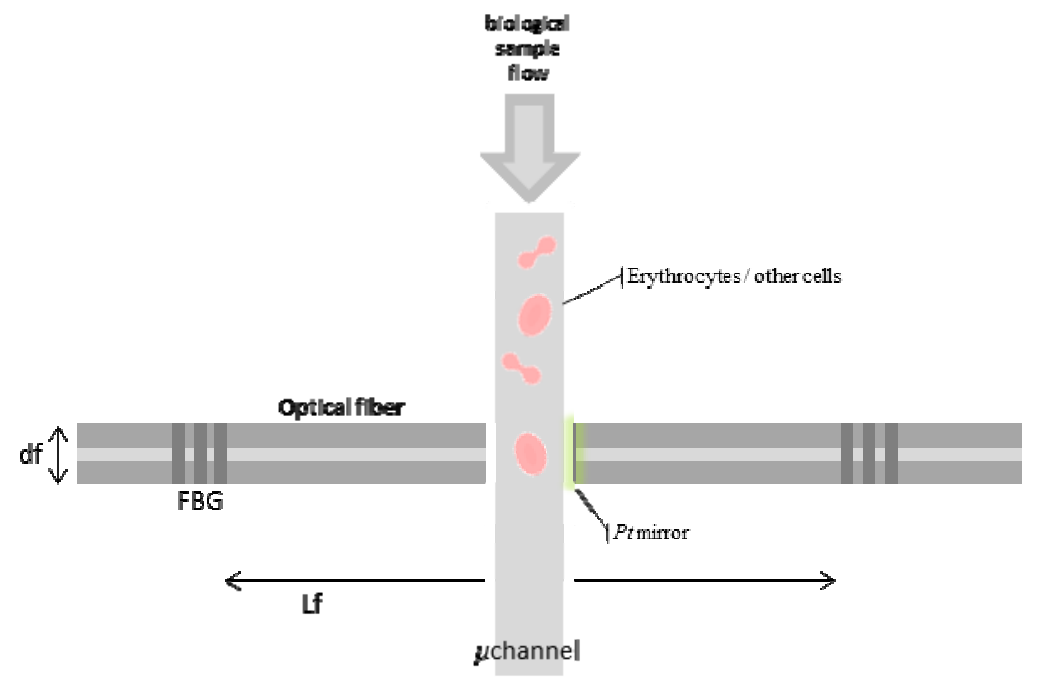

Figure 2. Schematic representation of the detection area of the SU-8 microchip. Different combinations are possible using FBG, SM or MM fiber and mirrored fiber tips.

\section{EXPERIMENTAL}

\subsection{Chip fabrication and characterization}

Float glass substrates $(0.55 \mathrm{~mm}$ thick; Guinchard, Switzerland) were covered with a thin layer of SU-8 (GM1060, Gersteltec, Switzerland) to improve adhesion between thick SU-8 structure and the glass substrate. Then, a $130 \mu \mathrm{m}$ thick SU-8 layer (GM1070, Gersteltec, Switzerland) was spin coated at $1060 \mathrm{rpm}$ for $40 \mathrm{~s}$ and soft baked. The samples were exposed to UV for dose of $550 \mathrm{~mJ} / \mathrm{cm}^{2}$ (MA6, Suss MicroTec, Germany) through i-line filter (G180336, Suss MicroTec, Germany) and post baked. Subsequently, the photoresist was developed for $9 \mathrm{~min}$ in propylene glycol methyl ether acetate (Sigma-Aldrich, Germany), rinsed and hard baked.

PDMS slab (Sylgard 184, Dow Corning, Spain) was used as a cover to seal from the top the SU-8 microchannels and insertion grooves.

The samples were characterized by Scanning Electron Microscope (Zeiss Auriga, Germany).

\subsection{Optical sensors optimization}

Several configurations for refractive index measurement based in on-chip fiber Fabry-Perot cavities were investigated and evaluated. Parameters such as the quality of the interferometric pattern, with a reasonable power budget, good fringe visibility and finesse factor were considered. Some constrains had to be considered because in order to enable simultaneous detection of refractive index and absorption/reflectance it was necessary to apply partially reflective coatings on a MM fiber tip and combine it with a SM fiber with an inscribed FBG for NIR measurements. Therefore, fringe visibility and efficiency of the acquisition of the scattered light required some compromise in the mirror and FBG reflectivity. At this stage, such tests were performed outside the chip using a microscope and precision alignment stages to implement the characterization and optimization of the FP cavity. 
Fiber Bragg grating structures with reflectivity ranging from $\mathrm{R} \approx 20 \%$. to $\mathrm{R} \approx 90 \%$, with a central wavelength around $1550 \mathrm{~nm}$, were inscribed in standard SMF-28 single mode fiber with a germanosilicate core (3 mol\%), previously hydrogenated at $100 \mathrm{~atm}$. The FBG was written using a $10 \mathrm{~mm}$ length diffractive phase mask $(\Lambda=1062 \mathrm{~nm})$, illuminated with a $\mathrm{KrF}$ laser $(248 \mathrm{~nm})$. Exposure time and laser energy were adjusted to obtain the desired reflectivity.

Multimode fibers were mirrored by RF-sputtering of a Palladium target. Reflectivity was controlled by adjusting the thickness of the coating layer.

It was observed that when high reflectivity was applied in both the mirror and the FBG a very high visibility and finesse were obtained, resulting in very sharp spectral lines, but with a poor signal-to-noise ratio in face of the little amount of power reaching the detector. The best results were achieved for an approximate reflectivity of $\sim 60 \%$ where a good compromise between fringe visibility and transmitted/reflected power could be observed.

Figure 3 presents the reflected interferometric FP spectrum obtained when a SM fiber with a FBG having an approximate reflectivity of $60 \%$ was aligned with a MM fiber with a partially reflective palladium layer with similar reflectivity (thickness $\mathrm{d} \sim 30 \mathrm{~nm}$ ). A visibility of approximately $45 \%$ was estimated from the acquired spectrum that is adequate for sensitive refractive index measurements. The spectrum was acquired in reflection through the SM fiber using a tunable laser and an optical circulator. As expected, the interferometric pattern follows a quasi-sinusoidal behavior modulated by the envelope of the FBG reflectivity function.

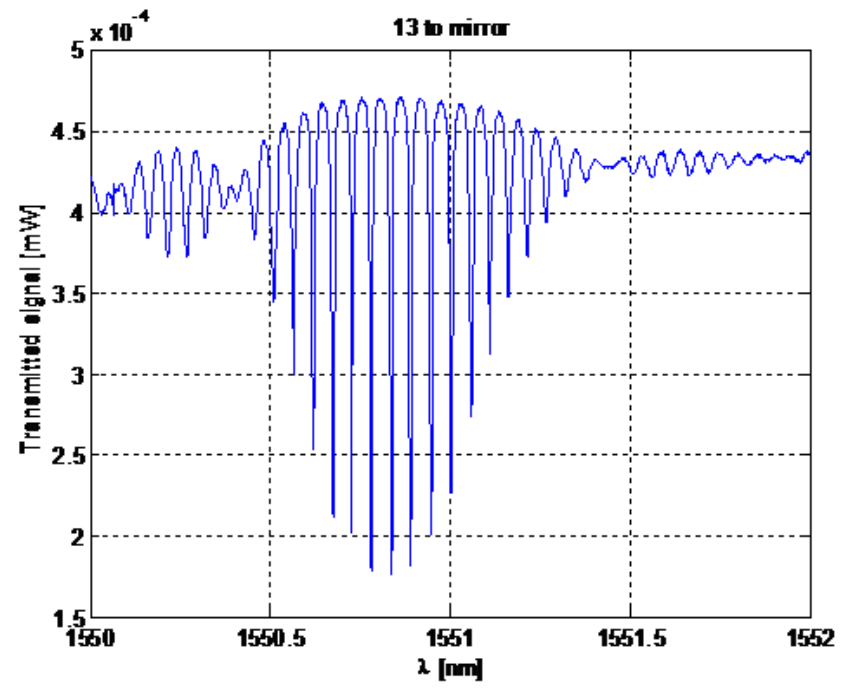

Figure 3: Interferometric spectrum of the FBG-mirrored fiber FP cavity with $\sim 60 \%$ reflectivity.

The MM fiber on the other hand was plugged into a USB4000 CCD Spectrometer (Ocean Optics, Inc., FL, USA). It was verified that for gaps between the MM and SM fiber in the range of $20 \mu \mathrm{m}$ a good signal-to-noise ratio could be obtained using standard halogen lamp for microscope illumination and CCD integration times ranging hundreds of ms, enabling the spectral measurements to be performed accurately.

\section{RESULTS}

\subsection{On-chip results}

The optical fibers were used to carry the radiation from the light source to the microchannel and then to the CCD spectrometer or to a photodetector. This approach allows guiding the light very close to the sample to be analyzed (minimum tested distance: $10 \mu \mathrm{m}$ ). The optical fibers and the microchannels are self-aligned in the microchip by the SU-8 features, therefore no fine alignment using micropositioners was needed. 
For the implementation of the channels and grooves, high aspect ratio SU-8 structures of smooth and nearly vertical side walls (figure $4 \mathrm{a}$ ) have been produced. The inserted fibers in the grooves are then self-aligned with good accuracy as can be observed from the Scanning electron micrographs in figure $4 \mathrm{~b}$.

Insertion of SM and mirrored MM fibers was straightforward and the transmission/reflection spectra observed had identical characteristics to those obtained previously using precision translation stages demonstrating the viability of the dual measurement concept inside the microfluidic chip.

\subsection{Optical measurements}

For preliminary tests of simultaneous absorption and refractive index measurements of biological samples tests were performed using the fiber optic cavities aligned outside the chip using three sample solutions with the same volume and with different refractive index and absorption properties.

For reference purposes a pure water solution was used (solution 1). In addition two samples of Acid Red 37dye in ethanol were diluted into pure water. Solution 2, having $25 \%$ of dye, and solution 3, having $50 \%$ of dye, were respectively probed. Refractive index should vary roughly from 1.333 (water) nearly up to 1.36 (ethanol), while absorption bellow $\sim 640 \mathrm{~nm}$ was expected to increase with dye concentration.

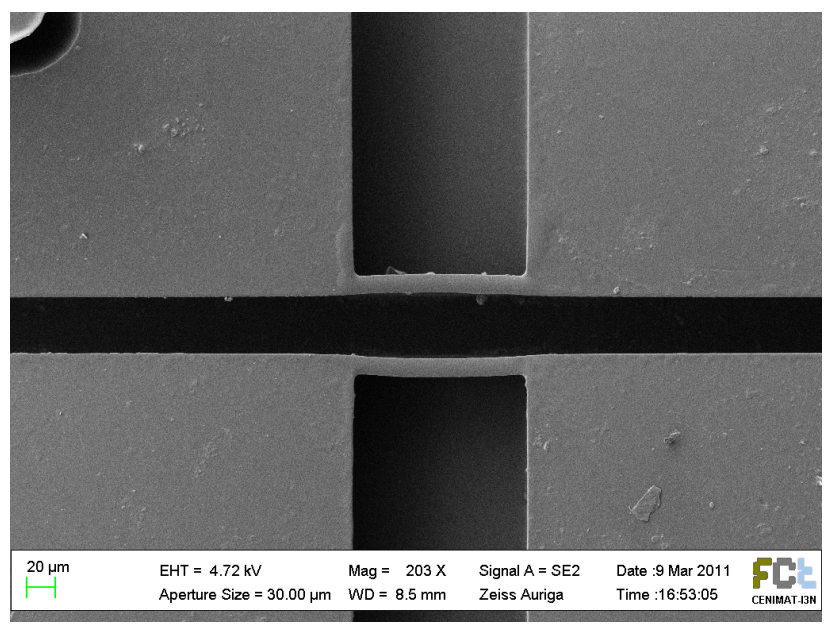

(a)

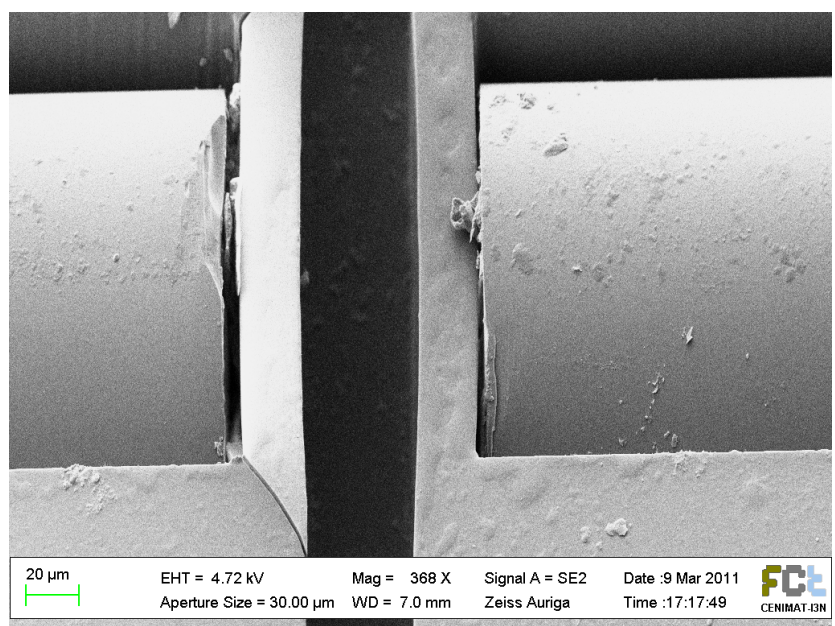

(b)

Figure 4. Scanning electron micrographs of microchannel and grooves for the fibers insertion: (a) without optical fibers and (b) with inserted optical fibers.

The test solutions were characterized using the setup represented in figure 5. The FP cavity used was based on a $60 \%$ reflectivity FBG, with $1 \mathrm{~nm}$ wide spectral bandwidth, and MM fiber tip coated with a $30 \mathrm{~nm}$ mirror. The cavity is divided into two main parts - in-fiber cavity with constant optical path, and a detection area corresponding to the channel of changing optical path. The length of the fiber cavity was $\mathrm{L} \sim 15 \mathrm{~mm}$ with refractive index of $\mathrm{n}_{\text {core }}=1.458$. The detection area with changing refractive index was restricted to the spacing between the fibers which was set to $\sim 30 \mu \mathrm{m}$. The SM fiber was illuminated by an Erbium doped broad-band optical source through an optical circulator that redirected the reflected signal towards an optical spectrum analyzer for detection. On the other side of the gap, the mirrored MM fiber was plugged into the CCD spectrometer for visible-NIR detection, while the fiber gap was illuminated from above with a halogen lamp. 


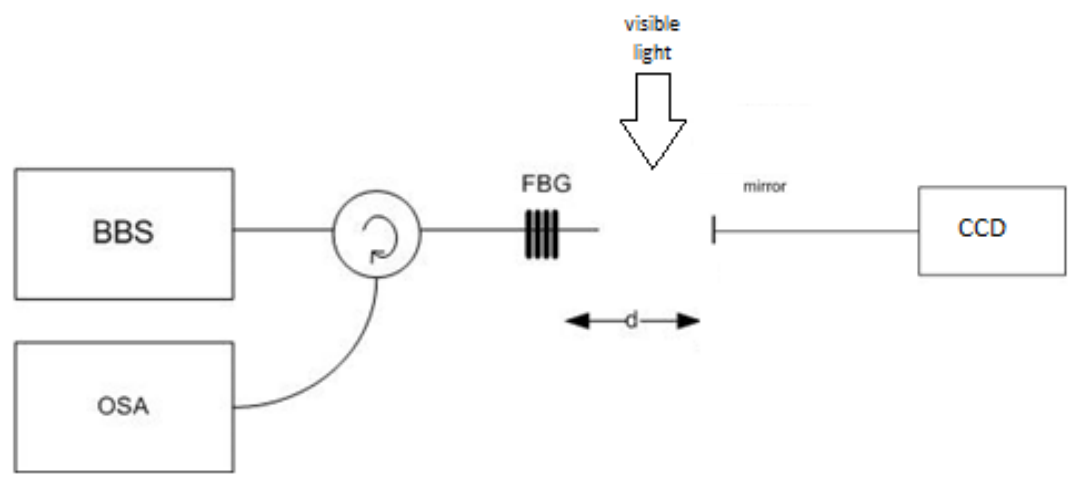

Figure 5: Measuring setup for simultaneous assessment of refractive index and spectroscopy characteristics.

Acquired spectra are presented in figure 6a (full span of the FBG) and $6 \mathrm{~b}$ (detail of a wavelength period). Clear spectral shifts can be observed in the interferometric pattern that can be related with the solutions refractive index.

Knowing the parameters of the sensing cavity, the wavelength shift can be easily converted into a phase shift and the relative refractive index changes can then be calculated. Results of the estimated refractive indexes are presented in table 1. The calibration of the system was performed by using the reference spectrum of the known refractive index solution (solution 1). The results obtained clearly follow the expected tendency where an increase is observed from pure water $(1,333)$ to water with dye and approximately $50 \%$ ethanol $(1,3433)$.

\begin{tabular}{|c|c|c|}
\hline Solution & $\Delta \lambda(\mathrm{pm})$ & $\mathrm{n}_{1}$ \\
\hline 1 & 0,00 & 1,333 \\
\hline 2 & 7,00 & 1,3332 \\
\hline 3 & 29,50 & 1,3433 \\
\hline
\end{tabular}

Table 1: Spectral sifts observed and corresponding calculated refractive index.
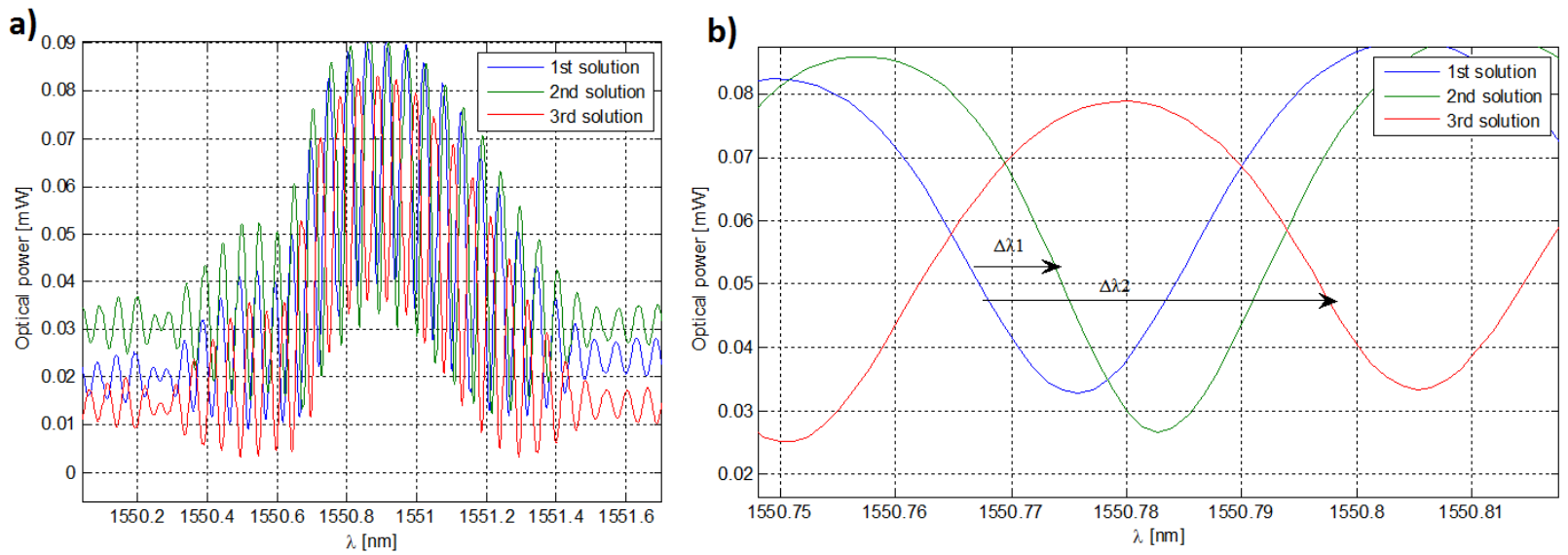

Figure 6: The spectra of FP cavity for pure water (first solution), water with $25 \%$ dye in ethanol (second solution), and water with $50 \%$ dye in ethanol (third solution). a) Full span of FBG spectrum; b) Detail of wavelength shift between acquired spectra.

Using the MM detection fiber, the color changes induced by the presence of the dye were quite noticeable as shown in figure 7. For pure water all the spectrum of the halogen lamp could be observed unchanged. However, for the solution with added dye the transmission was significantly reduced for wavelengths shorter than $640 \mathrm{~nm}$. For the third solution with higher dye concentration the reduction of transmission was significantly larger. 


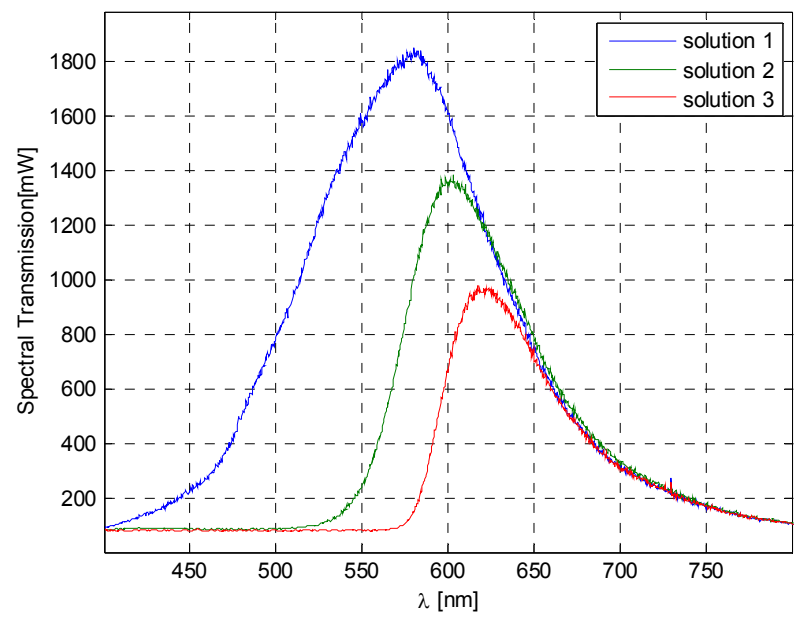

Figure 7: Recorded visible spectra, collected by the MM fiber, of the solutions having different concentrations of red dye.

The results obtained clearly indicate the viability of performing simultaneously the analysis of the refractive index properties as well as the spectroscopic characteristics of complex samples contributing for more advanced tools for single cell analysis when combined with microfluidic platforms.

\section{CONCLUSIONS}

In this paper we presented a microfluidic chip with an optical configuration for simultaneous determination of refractive index and reflectance/absorbance spectra suitable for analysis of biological samples. Different fiber optic configurations and parameters were tested in order to optimize the detection methods for refractive index and reflectance measurements.

Preliminary results showed the viability of fabricating chips with high aspect ratio features using an SU-8 platform where optical fibers could be self-aligned. Tests performed showed the viability of simultaneous evaluation of the color and refractive index characteristics of complex samples demonstrating the viability of the concept.

\section{ACKNOWLEDGEMENTS}

This work was supported by "Fundação para a Ciência e Tecnologia" (PTDC/SAU-BEB/102247/2008, Hybrid) and a PhD scholarship of reference SFRH/BD/44258/2008.

\section{REFERENCES}

[1] Kuswandi, B., Nuriman, Huskens, J. and Verboom, W. (2007). Optical sensing systems for microfluidic devices: A review. Analytica Chimica Acta 601(2): 141-155.

[2] Fan, X.D., et al., Sensitive optical biosensors for unlabeled targets: A review. Analytica Chimica Acta, 2008. 620(1-2): p. 8-26.

[3] Liang, X.J., Zhang, X.M., Ni, N., Liu, A.Q., An approach of cancer cells diagnosis using biophotonic chip, Proceedings of SPIE - The International Society for Optical Engineering 5994, art. no. 599404

[4] Park, Y., Diez-Silva, M., Popescu, G., Lykotrafitis, G., Choi, W., Feld, M., and Suresh, S. Refractive index maps and membrane dynamics of human red blood cells parasitized by Plasmodium falciparum. PNAS. September 16, 2008. vol. 105 no. 37. pp13730-13735. 
[5] Manz, A., Graber, N. and Widmer, H. M. (1989). "Miniaturized Total Chemical-Analysis Systems - a Novel Concept for Chemical Sensing”. 5th International Conf on Solid-State Sensors and Actuators and Eurosensors 3 ( Transducers 89 ), Elsevier Science Sa Lausanne. Montreux, Switzerland

[6] Abgrall, P., \& Gué, a-M. (2007). Lab-on-chip technologies: making a microfluidic network and coupling it into a complete microsystem-a review. Journal of Micromechanics and Microengineering, 17(5), R15-R49

[7] Bashir, R. (2004). BioMEMS: state-of-the-art in detection, opportunities and prospects. Advanced drug delivery reviews, 56(11), 1565-86..

[8] Young, E. W. K and Beebe, D. (2010) "Fundamentals of microfluidic cell culture in controlled microenvironments" Chemical Society Reviews, Vol. 39, pp. 136-148.

[9] Nascimento, E., Nogueira, N., Silva, T., Braschler, T., Demierre, N., Renaud, P., Oliva, A. (2008) Dielectrophoretic sorting on a microfabricated flow cytometer: label free separation of Babesia bovis infected erythrocytes. Bioelectrochemistry Volume 73, Issue 2, Pages 123-128.

[10] Campo, a D., \& Greiner, C. (2007). SU-8: a photoresist for high-aspect-ratio and 3D submicron lithography. Journal of Micromechanics and Microengineering, 17(6), R81-R95.

[11] LK Chin, AQ Liu, CS Lim, PH Yap, Multiphase flow manipulation for continuous refractive index analysis of single living cell, Eleventh International Conference on Miniaturized Systems for Chemistry and Life Sciences $7-11$ October 2007, Paris, FRANCE.

[12] Song, W.Z., Zhang, X.M., Liu, A.Q., Lim, C.S., Yap, P.H., Hosseini, H.M.M., Refractive index measurement of single living cells using on-chip Fabry-Pérot cavity, Applied Physics Letters 89 (20), art. no. 203901

[13] Chin, L.K., Liu, A.Q., Lim, C.S., Zhang, X.M., Ng, J.H., Hao, J.Z., Takahashi, S., Differential single living cell refractometry using grating resonant cavity with optical trap, Applied Physics Letters 91 (24), art. no. 243901

[14] Serebrennikova, Y., Smith, J., Huffman, D., Leparc, G., García-Rubio, L.,(2008) Quantitative interpretations of Visible-NIR reflectance spectra of blood.. Vol. 16, No. 22. Optics Express. pp18216. 